\title{
I solation and polymerase chain reaction-based identification of Riemerella anatipestifer from ducks in Kerala, India
}

\author{
Manju Soman, Sreeja R. Nair, M. Mini, Binu K. Mani and Siju J oseph
}

Department of Veterinary Microbiology, College of Veterinary and Animal Sciences, Mannuthy, Thrissur, Kerala, India. Corresponding author: Manju Soman, e-mail: manjuso1993@gmail.com, SRN: drsreejarnair@gmail.com, MM: drmmini@yahoo.co.in, BKM: binukmani@yahoo.com, SJ: siju96@gmail.com

Received: 29-06-2014, Revised: 27-08-2014, Accepted: 01-09-2014, Published online: 05-10-2014

doi: 10.14202/vetworld.2014.765-769. How to cite this article: Soman M, Nair SR, Mini M, Mani BK, Joseph S (2014) Isolation and polymerase chain reaction-based identification of Riemerella anatipestifer from ducks in Kerala, India, Veterinary World 7(9): 765-769.

\begin{abstract}
Aim: The aim was to isolate and characterize Riemerella anatipestifer organisms from disease outbreaks in ducks in Kerala.

Materials and Methods: Ducklings, suspected of Riemerella infection, were sacrificed and subjected to post-mortem examination. Heart blood smears and impression smears from liver and spleen were examined for the presence of pathogenic organisms. Heart blood, lung, liver, and spleen collected aseptically from the birds were subjected to isolation trials in brain heart infusion agar and 10\% bovine blood agar. The isolates were characterized based on morphology, cultural characteristics and biochemical tests, and their identity were confirmed by polymerase chain reaction (PCR) and the PCR amplified DNA was sequenced. The antibiotic sensitivity testing of the isolates were carried out using six antibiotics viz ciprofloxacin, chloramphenicol, enrofloxacin, amoxycillin, cotrimoxazole, and gentamicin.
\end{abstract}

Results: Colonies suggestive of Riemerella organisms could be isolated on blood agar. Biochemical characterization and PCR confirmed the identity of isolates as $R$. anatipestifer. The nucleotide sequence of the PCR product showed $99 \%$ homology to the $R$. anatipestifer sequences in the NCBI. The antibiogram revealed that the organisms were sensitive to ciprofloxacin, enrofloxacin, and gentamicin.

Conclusion: The present study suggests that the PCR assay can facilitate fast and proper identification of R. anatipestifer infection in ducks. The assay can also differentiate between $R$. anatipestifer and Pasteurella multocida and can replace the traditional methods of differentiation which are cumbersome and time-consuming.

Keywords: antibiogram, ducks, isolation, polymerase chain reaction, Riemerella anatipestifer.

\section{Introduction}

Riemerella anatipestifer is a major bacterial pathogen of avian species, causing severe economic loss in commercially important poultry worldwide. The disease is commonly referred as "new duck disease" and variously known as infectious serositis, duck septicaemia and anatipestifer syndrome. Affected ducks, usually 1-7 weeks of age, often develop ocular and nasal discharges, mild coughing and sneezing, tremors of the head and neck, and incoordination. In typical cases, affected ducklings may lie on their back with paddling movement of legs. Necrotic dermatitis on the lower back or around the vent may also be observed.

Mortality is usually 2-50\%. Fibrinous polyserositis, pericarditis, perihepatitis, and airsacculitis are commonly observed as the gross post-mortem lesions. Infection of the central nervous system can result in fibrinous meningitis. Birds with chronic form of the disease may develop mucopurulent or caseous salpingitis leading to loss of egg production [1]. The large scale mortality inflicted by this disease has become a cause of major concern in commercial duck farmers in Kerala.

Copyright: The authors. This article is an open access article licensed under the terms of the Creative Commons Attributin License (http:// creative commons.org/licenses/by/2.0) which permits unrestricted use, distribution and reproduction in any medium, provided the work is properly cited.
The present study reports the isolation, identification, and antibiogram of $R$. anatipestifer from different incidence of disease outbreak in Kerala.

\section{Materials and Methods \\ Ethical approval}

All the procedures have been carried out in accordance with the guidelines laid down by Institutional Ethics Committee and in accordance with local laws and regulations.

During the months of April and May 2013, heavy mortality in ducklings of age group of 4-8 weeks was reported from Central Kerala. The ducklings received for examination were designated as D1, D2, and D3. They showed signs of respiratory distress and neurologic signs such as trembling of head and neck, ataxia, and heavy mortality.

The ducklings were sacrificed and subjected to post-mortem examination. Heart blood smears and impression smears from liver and spleen were stained by Leishman's stain and examined for the presence of pathogenic organisms. Samples of heart blood, lung, liver, and spleen collected aseptically from the birds were used for isolation of the organism. The clinical samples were directly streaked on MacConkey agar, Brain heart infusion agar (BHIA) and 10\% bovine blood agar. MacConkey agar and BHIA were incubated for $24 \mathrm{~h}$ at $37^{\circ} \mathrm{C}$ under ordinary conditions while 
blood agar was incubated at $37^{\circ} \mathrm{C}$ for $24 \mathrm{~h}$ in an atmosphere enriched with 5\% CO. The samples were also inoculated on Sabouraud's dextrose agar (SDA) and incubated at room temperature for 7 days.

Characterization of the isolate was done based on morphology, cultural characters, and biochemical tests [2].

Ducklings in the age group of 9-10 days and mice in the age group of 6 weeks were subjected to pathogenicity testing using the isolates [3]. Mice were injected with $0.2 \mathrm{ml}$ of inoculum with a concentration of $3 \times 10^{8}$ organisms/ml intraperitoneally. The ducklings were given $1 \mathrm{ml}$ of inoculum with the same concentration of organism by intramuscular route. Two control animals were kept for each experiment. The mice and birds were observed for the development of clinical signs or death for a period of 2 weeks. Re-isolation of the organism was attempted from heart blood, lung, liver, spleen, and brain in 10\% bovine blood agar.

Polymerase chain reaction (PCR) was performed to confirm the identity of the isolates as per Kardos et al. [4] with minor modifications. Template DNA was extracted by the method of heat lysis of the bacterial culture. Pure culture in $5 \mathrm{ml}$ of phosphate buffered saline (PBS) was centrifuged at $3000 \times g$ for $10 \mathrm{~min}$ at $4^{\circ} \mathrm{C}$, and the pellet was washed twice in PBS. The final pellet was resuspended in $100 \mu$ l of triple distilled water. It was boiled for $10 \mathrm{~min}$ and chilled in ice for $30 \mathrm{~min}$. After centrifugation at $3000 \times \mathrm{g}$ for $5 \mathrm{~min}$ at $4^{\circ} \mathrm{C}$, the supernatant was collected and used as template DNA. PCR was performed in a $25 \mu$ reaction mixture. Riemerella specific primers, forward primer 5'-TTACCGACTGATTGCCTTCTAG-3' and reverse primer 5'-AGAGGAAGACCGAGGACATC-3' were used in the study. To each PCR tube, $20 \mu \mathrm{l}$ of master mix containing 20 pmol of each primer, $200 \mu \mathrm{M}$ of each dNTP, one unit of Taq DNA polymerase in a PCR reaction buffer with $50 \mathrm{mM} \mathrm{KCl,} 10 \mathrm{mM}$ Tris $\mathrm{HCl}, 1.5 \mathrm{mM} \mathrm{MgCl}$, and $5 \mu \mathrm{l}$ of template DNA were added. One negative control without template DNA was included to monitor contamination.

PCR was carried out in Applied Biosystems thermal cycler with an initial denaturation at $95^{\circ} \mathrm{C}$ for $4 \mathrm{~min}, 35$ cycles of $95^{\circ} \mathrm{C}$ for $1 \mathrm{~min}, 55^{\circ} \mathrm{C}$ for $1 \mathrm{~min}$, and $72^{\circ} \mathrm{C}$ for 1 min followed by a final extension of $72^{\circ} \mathrm{C}$ for $7 \mathrm{~min}$ The amplified PCR products were electrophoresed in 1\% agarose gel for $1 \mathrm{~h}$ at $50 \mathrm{~V}$.

The amplified DNA was sequenced from SciGenom, Cochin, Kerala, India.

The antibiotic sensitivity test, of the isolates, was performed using six antibiotics viz ciprofloxacin, chloramphenicol, enrofloxacin, amoxycillin, co-trimoxazole, and gentamicin [5].

\section{Results}

Post-mortem lesions of fibrinous polyserositis, fibrinous pericarditis, perihepatitis, and airsacculitis were observed in D1 and D2. In addition to this, D3 revealed the presence of esophageal corrugations suggestive of duck plague.

Heart blood smears and impression smears from liver and spleen revealed the presence of bipolar stained organisms.

The blood agar plates yielded confluent, grey, moist, convex, entire, butyrous, transparent, non-hemolytic dew drop colonies in $24 \mathrm{~h}$ (Figure-1). No growth was observed on BHIA, MacConkey agar and SDA. Gram's staining of the colonies revealed small, Gram-negative organisms with a morphology varying from cocco-bacillary, short rods to filamentous forms. The organisms were found to be nonmotile, and were catalase and oxidase positive. Results of biochemical tests showed that the isolate was negative for indole, methyl red, Voges-Proskauer, citrate and nitrate tests and positive for urease test.

The mice subjected to pathogenicity tests, remained healthy even after a period of 14 days and $R$. anatipestifer could not be re-isolated from these animals. All the duckling subjected to pathogenicity tests, died within 12-48 h while the control duckling remained healthy. The gross lesions observed were enlargement and congestion of the liver and enlarged spleen. Heart blood and liver impression smears revealed bipolar organisms when stained with Leishman's stain. $R$. anatipestifer could be re-isolated from heart blood, lung, liver, and spleen of the dead duckling's on 10\% bovine blood agar and its identity was confirmed by PCR.

The PCR products revealed an amplicon size of 546 bp when viewed under gel documentation system (Figure-2).

Nucleotide sequence of the amplified product showed 99\% homology to that of $R$. anatipestifer sequences deposited in the GenBank, NCBI (Figures-3 and 4 ).

Antibiotic sensitivity test revealed that the isolates were sensitive to ciprofloxacin, enrofloxacin, and gentamicin and were resistant to chloramphenol, amoxycillin, and co-trimoxazole.

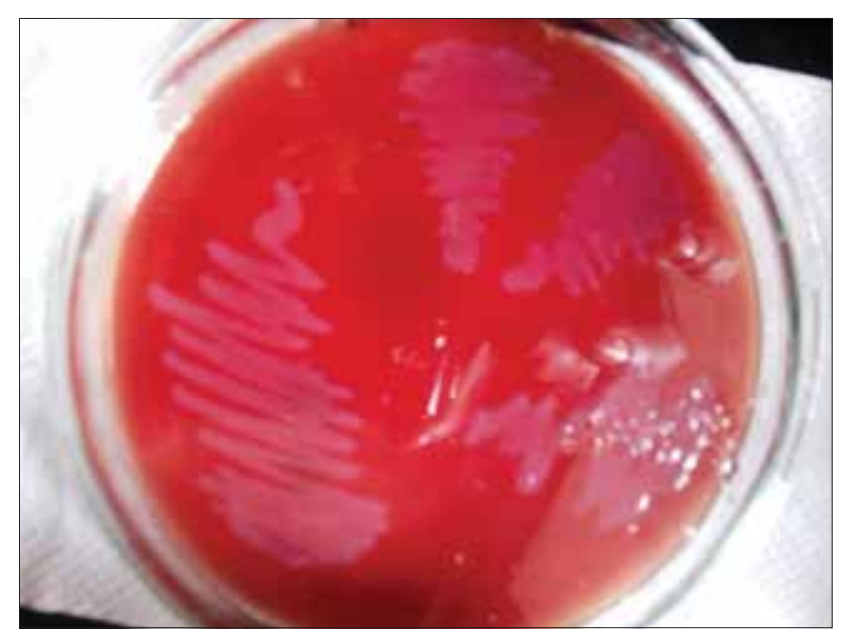

Figure-1: Blood agar plate showing Riemerella anatipestifer isolates. 


\section{Discussion}

$R$. anatipestifer infection is often confused with duck pasteurellosis, and hence an accurate and early diagnosis of this infection is important to avoid heavy loss by mortality. Identification based on cultural and biochemical characteristics is time-consuming and laborious and as Riemerella is characterized by the absence of certain specific phenotypic properties, identification by phenotypic properties or biochemical characteristics alone is difficult. The PCR is a rapid, sensitive and highly specific assay for detection of microbial infections. In this study, we used PCR to confirm the identity of the isolates obtained. The same could be used to detect Riemerella organisms directly on clinical specimens, and thus help in implementing

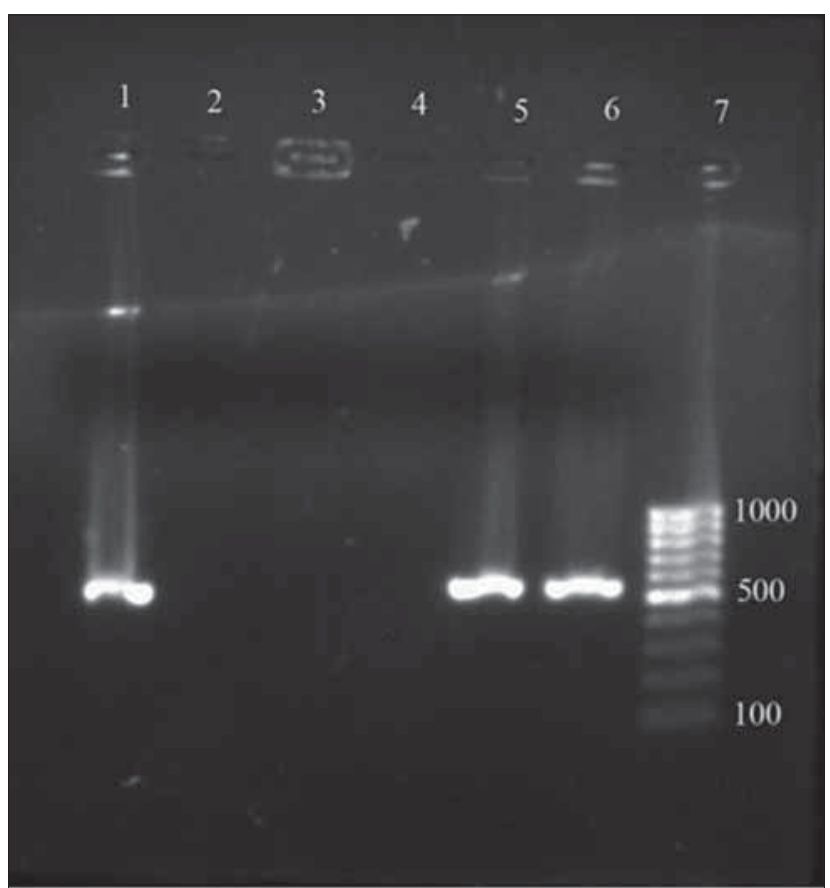

Lane 1-D1， Lane 5-D2, Lane 6-D3

Lane 7-100 bp DNA marker

Figure-2: Riemerella anatipestifer-Polymerase chain reaction amplified DNA on agarose gel. early treatment and control strategies. In this study, a co-infection of duck plague and $R$. anatipestifer was detected in one of the ducklings. An immunosuppressive state induced by duck plague may explain the presence of secondary infections of Pasteurella multocida, R. anatipestifer and Escherichia coli in natural outbreaks of duck plague in ducklings [1].

The primers used in the study were selected [4]. A 700 bp fragment of DNA, common to 16 Riemerella isolates, was amplified using enterobacterial repetitive intergenic consensus PCR (ERIC PCR), sequenced and used to design a primer for a PCR which could amplify a 546 bp conserved sequence of $R$. anatipetifer [4]. They reported that the PCR assay was specific for $R$. anatipestifer and was capable of correctly identifying the organism from pure culture as well as from clinical samples and could differentiate between $R$. anatipestifer and P. multocida. A PCR assay for detection of a conserved region of the $16 \mathrm{~S}$ rRNA gene of $R$. anatipestifer, was developed [6], which could successfully detect the organism in clinical specimens. A new Riemerella-specific PCR assay was designed by Rubbenstroth et al. [7] who also conducted matrix-assisted laser desorption/ionization-time-of-flight mass spectrometry fingerprinting of whole bacterial cells to identify Riemerella isolates. In a study conducted to determine the gene profile of $R$. anatipestifer organisms, [8] detected a total of 48 genes in the Riemerella genome of which eight were novel genes which were thought to be potent virulence factors. A multiplex PCR assay for simultaneously detecting four pathogenic bacteria in ducks viz, P. multocida, Salmonella enterica, $R$. anatipestifer, and $E$. coli was developed using target genes KMT1 of $P$. multocida, the invasion protein gene of $S$. enterica, 16S rDNA of $R$. anatipestifer, and the alkaline phosphatase gene of $E$. coli. The assay could detect the four bacteria in laboratory as well as field samples [9]. In the present study PCR could successfully amplify $R$. anatipestifer DNA and this was confirmed by nucleotide sequencing.

More than 20 different serotypes of $R$. anatipestifer have been reported to cause infection in

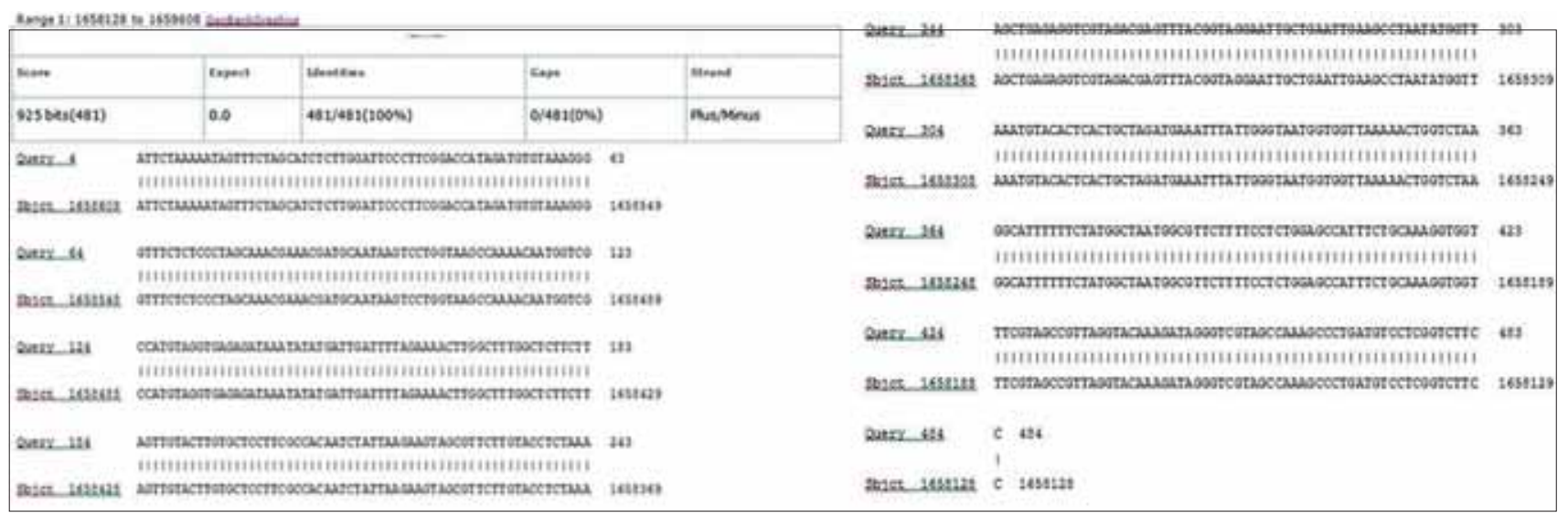

Figure-3: Sequence analysis of the isolate of Riemerella anatipestifer with R. anatipestifer RA-CH-2, complete genome, published in NCBI. 


\begin{tabular}{|c|c|c|c|c|c|c|}
\hline Description & $\underset{\text { score }}{\text { Max }}$ & $\begin{array}{l}\text { Total } \\
\text { score }\end{array}$ & $\begin{array}{l}\text { Query } \\
\text { cover }\end{array}$ & $\begin{array}{c}E \\
\text { value }\end{array}$ & Ident & Accession \\
\hline 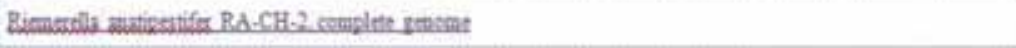 & 925 & 925 & $99 \%$ & 0.0 & $100 \%$ & CPOQtover \\
\hline 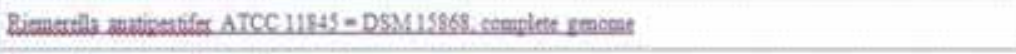 & 925 & 925 & $99 \%$ & 0.0 & $100 \%$ & CP0033881 \\
\hline 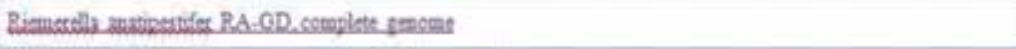 & 925 & 925 & $99 \%$ & 0.0 & $100 \%$ & C2002421 \\
\hline 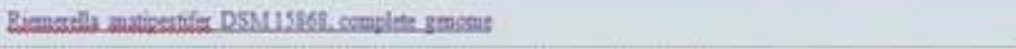 & 925 & 925 & $99 \%$ & 0.0 & $100 \%$ & cPosez:461 \\
\hline 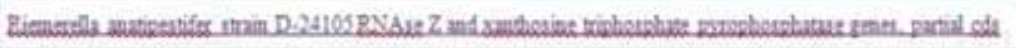 & 892 & 892 & $95 \%$ & 0.0 & $100 \%$ & 2N5732321 \\
\hline
\end{tabular}

Figure-4: The NCBI blast search analysis of the sequenced DNA of field isolate of Riemerella anatipestifer with the published sequences.

field cases. A comparative genomic analysis of three Riemerella isolates demonstrated the genetic diversity between the isolates [10]. Inactivated bacterins prepared from different serotypes of $R$. anatipestifer do not confer cross-protection to other serotypes. This lack of cross-protection limits the usefulness of vaccination against this disease. Consequently, chemotherapy is very important aspect in the treatment of ducks infected with $R$. anatipestifer [11]. A study conducted [12], showed levamisole to be a useful adjuvant in inactivated Riemerella vaccine and that it could enhance the immune response of ducklings and also alleviate local injection lesions by reducing the dose of the vaccine. A trivalent inactivated vaccine of $R$. anatipestifer, including serotype 1,2 , and 10 showed $100 \%$ protection in experimental animals from challenge with any of the three serotypes [13]. In vitro and in vivo antimicrobial susceptibilities of 50 field duck isolates of $R$. anatipestifer, to ceftiofur and 16 other commonly used antimicrobials was carried out [11]. They found that the Riemerella organisms were most sensitive to penicillin, ceftiofur, cephalothin, chloramphenicol, flumequine, and kanamycin. In another study conducted [14], R. anatipestifer was reported to be sensitive to enrofloxacin, chloramphenicol, lincomycin, streptomycin, and neomycin but resistant to penicillin $G$, ampicillin, tetracycline, trimethoprim-sulfamethoxazole, kanamycin, and gentamycin. Clonal spread of multi-drug resistant $R$. anatipestifer isolates among ducks in farms in China has been reported [15]. In the present study, the isolates were found to be sensitive to ciprofloxacin, enrofloxacin, and gentamicin and were resistant to amoxicillin, chloramphenicol, and co-trimoxazole.

\section{Conclusion}

The present study suggests that PCR assay will facilitate fast and proper identification of $R$. anatipestifer infection in ducks. Furthermore, the assay can easily differentiate between $R$. anatipestifer and $P$. multocida, and can replace the traditional methods of differentiation which is difficult and time-consuming. The ability of PCR assay to detect Riemerella organisms directly in clinical specimens is an added benefit. Confirmatory identification of $R$. anatipestifer infection in ducks in Kerala has laid the foundation for the development of an effective vaccine for this disease and in effect could provide economic relief to the commercial duck farmers in Kerala.

\section{Authors' Contribution}

MS and SRN carried out the study. MS drafted and revised the article. MM, BKM and SJ participated in scientific discussion. All authors read and approved the final manuscript.

\section{Acknowledgments}

The authors are highly thankful to the Dean, College of Veterinary and Animal Sciences, Mannuthy, for providing facilities and fund for this study.

\section{Competing I nterests}

The authors declare that they have no competing interests.

\section{References}

1. Kahn, C.M., editor. (2010) Riemerella anatipestifer infection. The Merck Veterinary Manual. $10^{\text {th }}$ ed. Merck and Co Publishers, USA. p2945.

2. Quinn, P.J., Markey, B.K., Carter, M.E., Donnelly, W.J. and Leonard, F.C. (2002) Veterinary Microbiology and Microbial Diseases. Blackwell Sciences Ltd. 648p.

3. Curtis, P.E. (1985) Pasteurella multocida In: Collins C.H. and Grange J.M., editors. Isolation and Identification of Microorganisms of Veterinary Importance. Academic Press, London. p43.

4. Kardos, G., Nagy, J., Antal, M., Bistyak, A., Tenk, M. and Kiss, I. (2007) Development of a novel PCR assay specific for Riemerella anatipestifer. Lett. Appl. Microbiol., 44(2): 145-148.

5. Bauer, A.W., Kirby, W.M., Sherris, J.C. and Turck, M. (1966) Antibiotic susceptibility testing by a standardized single disc method. Am. J. Clin. Pathol., 45(4): 493-496.

6. Qu, F.F., Cai, C., Zheng, X.J. and Zhang, D.B. (2006) Rapid identification of Riemerella anatipestifer on the basis of specific PCR amplifying 16S rDNA. Wei. Sheng. Wu. Xue. Bao., 46(1): 13.

7. Rubbenstroth, D., Ryll, M., Knobloch, J.K., Kohler, B. and Rautenschlein, S. (2013) Evaluation of different diagnostic tools for the detection and identification of Riemerella anatipestifer. Avian. Pathol., 42(1): 17-26.

8. Zhou, Z., Zheng, J., Tian, W., Li, J., Zhang, W., Zhang, J., Meng, X., Hu, S., Bi, D. and Li, Z. (2009) Identification of Riemerella anatipestifer genes differentially expressed in infected duck livers by the selective capture of transcribed sequences technique. Avian. Pathol., 38(4): 321-9.

9. Wei, B., Cha, S.Y., Kang, M., Park, I.J., Moon, O.K., Park, C.K. and Jang, H.K. (2013) Development and application of a multiplex PCR assay for rapid detection of 4 major bacterial pathogens in ducks. Poult. Sci., 92(5): 1164-70.

10. Wang, X., Liu, W., Zhu, D., Yang, L., Liu, M., Yin, S., 
Wang, M., Jia, R., Chen, S., Sun, K., Cheng, A. and Chen, X. (2014) Comparative genomics of Riemerella anatipestifer reveals genetic diversity. BMC. Genomics, 15(1): 479.

11. Chang, C.F., Lin, W.H., Yeh, T.M., Chiang, T.S. and Chang, Y.F. (2003) Antimicrobial susceptibility of Riemerella anatipestifer isolated from ducks and the efficacy of ceftiofur treatment. J. Vet. Diagn. Invest., 15(1): 26-29.

12. Zhang, Y., Chen, H., Zeng, X., Wang, P., Li, J. and Wu, W. (2014) Levamisole enhances immunity in ducklings vaccinated against Riemerella anatipestifer. Microbiol. Immunol., 58(8): 456-62.
13. Liu, H., Wang, X., Ding, C., Han, X., Cheng, A., Wang, S. and Yu, S. (2013) Development and evaluation of a trivalent Riemerella anatipestifer-inactivated vaccine. Clin. Vaccine. Immunol., 20(5): 691-7.

14. Zhong, C.Y., Cheng, A.C., Wang, M.S., Zhu, D.K., Luo, Q.H., Zhong, C.D., Li, L. and Duan Z. (2009) Antibiotic susceptibility of Riemerella anatipestifer field isolates. Avian. Dis., 53(4): 601-607.

15. Sun, N., Liu, J.H., Yang, F., Lin, D., Li, G., Chen, Z. and Zeng, Z. (2012) Molecular characterization of the antimicrobial resistance of Riemerella anatipestifer isolated from ducks. Vet. Microbiol., 158(3-4): 376-383.

$* * * * * * * *$ 